\title{
Anomalous inverse proximity effect in unconventional superconductor junctions
}

\author{
Shu-Ichiro Suzuki $\odot,{ }^{1}$ Takashi Hirai, ${ }^{2}$ Matthias Eschrig, ${ }^{3}$ and Yukio Tanaka ${ }^{1}$ \\ ${ }^{1}$ Department of Applied Physics, Nagoya University, Nagoya 464-8603, Japan \\ ${ }^{2}$ College of Engineering, Chubu University, Kasugai, 487-8501, Japan \\ ${ }^{3}$ Institute of Physics, University of Greifswald, Felix-Hausdorff-Strasse 6, 17489 Greifswald, Germany
}

(Received 11 December 2020; revised 20 September 2021; accepted 23 September 2021; published 1 December 2021)

\begin{abstract}
We investigate the effects of Andreev bound states due to the unconventional pairing on the inverse proximity effect of ferromagnet/superconductor junctions. Utilizing quasiclassical Eilenberger theory, we obtain the magnetization penetrating into the superconductor. We show that in a wide parameter range the direction of the induced magnetization is determined by two factors: whether Andreev bound states are present at the junction interface and the sign of the spin-mixing angle. In particular, when Andreev bound states appear at the interface, the direction of the induced magnetization is opposite to that without Andreev bound states. We also clarify the conditions under which an inverted induced magnetization appears. Analyzing this effect helps distinguishing the pairing symmetry of a superconductor.
\end{abstract}

DOI: 10.1103/PhysRevResearch.3.043148

\section{INTRODUCTION}

Superconductors (SCs) dominated by an exotic pairing interaction are often so-called unconventional superconductors (USCs), which break apart from the global phase symmetry one or more additional symmetries of the normal state. In a conventional SC, the electrons usually form Cooper pairs due to the retarded attractive effective interaction resulting from electron-phonon coupling [1]. On the other hand, a repulsive interaction like the Coulomb interaction in strongly correlated superconductors requires the order parameter to change sign on the Fermi surfaces, resulting in anisotropic pairings like for example the $d$-wave pairing in high- $T_{c}$ cuprate SCs and in heavy-fermion SCs.

The internal phase of the pair potential plays an important role in forming Andreev bound states (ABSs) [2-4]. At an interface of an USC, an ABS can be formed by the interference between incoming and outgoing quasiparticles where the two quasiparticles feel different pair potentials depending on the direction of motion [5-7]. Emergence of interface ABSs changes the properties of superconducting junctions such as the transport properties [5,8-40] and magnetic response [41-53].

The ABSs can drastically change the proximity effect as well. The proximity effect is the penetration of Cooper pairs into a normal metal $(\mathrm{N})$ attached to a SC [54]. The conventional proximity effect introduces a spectral gap in the density of states (DOS) of the N metal [55-59] (i.e., a so-called minigap), whereas a zero-energy peak (ZEP) in the DOS signifies the appearance of ABSs $[11,42,59,60]$. Together with ABSs,

Published by the American Physical Society under the terms of the Creative Commons Attribution 4.0 International license. Further distribution of this work must maintain attribution to the author(s) and the published article's title, journal citation, and DOI. odd-frequency Cooper pairs $[18,61,62]$ are known to be induced simultaneously. Odd-frequency pairs are demonstrated to show anomalous response to the vector [42-51,63] and Zeeman potentials [52,53].

When a SC is in contact with a magnetic material, another type of proximity effect occurs. In a ferromagnet/SC (F/SC) junction, the magnetization in the $\mathrm{F}$ penetrates into the $\mathrm{SC}$ on the length scale of the superconducting coherence length $\xi_{0}$. This is called the (magnetic) inverse proximity effect (IPE) [64-71]. The IPE has been studied for junctions of conventional SCs. It was first studied in a ballistic junction of a ferromagnetic insulator (FI) and an s-wave SC [64]. In this case, the induced magnetization is antiparallel to the magnetization in the FI. In junctions with a ferromagnetic metal (FM) instead of an FI, the induced magnetization is antiparallel in the diffusive limit [65], whereas it can be parallel in the ballistic limit $[66,67]$. The IPE in conventional SC structures has been observed by several experimental techniques, e.g., ferromagnetic resonance [72,73], nuclear magnetic resonance [68], and polar Kerr effect [69]. How anisotropic pairing in USCs affects the IPE, in contrast, has not been discussed so far. In particular, ABSs and corresponding odd-frequency pairs are expected to affect how the magnetization penetrates into the SC.

In this paper, we theoretically study the IPE in F/USC junctions utilizing the quasiclassical Green's function theory. We show that, when ABSs appear, the IPE induces a magnetization with the opposite sign compared to that in the F/conventional-SC junction (see Fig. 1). Using first a onedimensional model, we show that for a single quasiclassical trajectory the direction of the induced magnetization is determined by two factors: existence of ABSs and the sign of the spin-mixing angle. We also show that spin-singlet and spin-triplet pairs near the interface show a correspondence when $T \sim T_{c}$. Spin-triplet odd-frequency $s$-wave Cooper pairs induced at the interface of a ferromagnet/spin-triplet $p$-wave $\mathrm{SC}$ junction behave in the same way as spin-singlet $s$-wave pairs induced at the interface of a junction with an $s$-wave SC. 


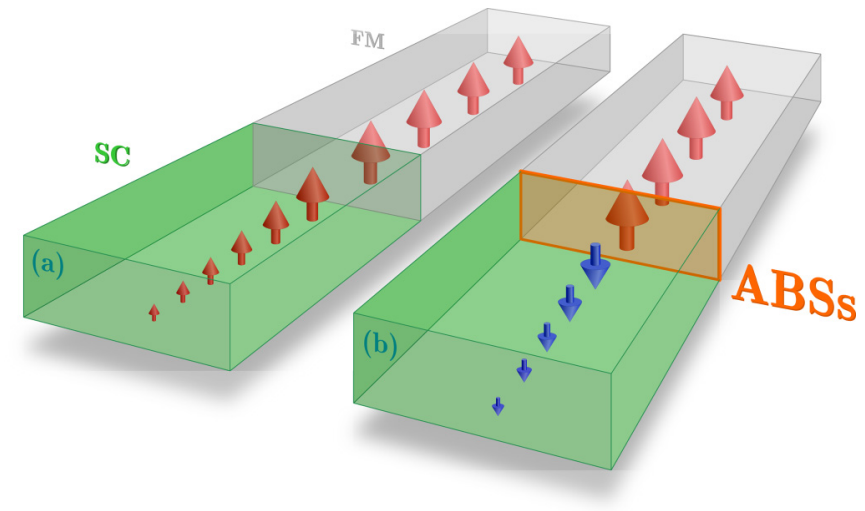

FIG. 1. Schematics of the inverse proximity effect (IPE). The arrows represent the magnetization vectors. The ABSs are in (a) absent and in (b) present at the interface. When there are ABSs, the magnetization induced in the superconductor (SC) is opposite to that without ABS. The length scale of the IPE is characterized by the superconducting coherence length $\xi_{0}$. The model system is twodimensional. The interface between the SC and the ferromagnet $(\mathrm{F})$ is aligned perpendicular to the $x$ - and parallel to the $y$ direction.

We then discuss a two-dimensional system, in which case the sign of the spin-mixing angle in general depends on the momentum parallel to the interface $k_{\|}$. When the magnetization in $\mathrm{F}$ is sufficiently small, the results are qualitatively the same as those in the $1 \mathrm{D}$ model. In the case of a large magnetization in $\mathrm{F}$, however, the $k_{\|}$dependence of the spinmixing angle cannot usually be ignored. As a result, in this latter case, the direction of the induced magnetization for superconductors with nodes at $k_{\|}=0$ is not simply determined by the two factors discussed in the $1 \mathrm{D}$ limit.

\section{MODEL AND FORMULATION}

We consider a ballistic superconducting junction as shown in Fig. 1, where the interface is located at $x=0$. The SC and F occupy $x \geqslant 0$ and $x<0$, respectively. The SC is modelled either as one-dimensional or two-dimensional. We discuss the magnetization induced at the interface of the $\mathrm{F} / \mathrm{SC}$ junction, where $\mathrm{F}$ and $\mathrm{SC}$ stand for ferromagnet and superconductor, respectively. In the IPE, the so-called spin-mixing angle $\theta_{\mathrm{SM}}$ plays an important role. We will introduce it when we discuss the boundary condition for the quasiclassical coherence function (see Sec. II B). An intuitive interpretation of $\theta_{\mathrm{SM}}$ is given in Appendix E.

\section{A. Quasiclassical Eilenberger theory}

Superconductivity in the ballistic limit can be described by the quasiclassical Eilenberger theory. The Green functions obey the Eilenberger equation:

$$
\begin{gathered}
i \boldsymbol{v}_{F} \cdot \nabla \check{g}+[\check{\mathcal{M}}, \check{g}]_{-}=0, \\
\check{g}=\left(\begin{array}{rr}
\hat{g} & \hat{f} \\
-\underset{\sim}{\hat{f}} & -\underset{\sim}{\hat{g}}
\end{array}\right), \quad \check{\mathcal{M}}=\left(\begin{array}{cc}
i \omega_{n} \hat{\sigma}_{0} & -\hat{\Delta} \\
-\underset{\sim}{\hat{\Delta}} & -i \omega_{n} \hat{\sigma}_{0}
\end{array}\right),
\end{gathered}
$$

where $\check{g}=\check{g}\left(x, \boldsymbol{k}, i \omega_{n}\right)$ is the Matsubara Green function, $\boldsymbol{v}_{F}$ is the Fermi velocity, $\omega_{n}=(2 n+1) \pi T$ is the Matsubara fre- quency, and $\hat{\Delta}$ is the pair-potential matrix. The accents $\check{`}$ and $\stackrel{\imath}{*}$ denote matrices in particle-hole space and spin space, respectively. The Pauli matrices in particle-hole space and in spin space are denoted $\check{\tau}_{j}$ and $\hat{\sigma}_{j}$ with $j \in\{1,2,3\}$, respectively, and the corresponding identity matrices by $\check{\tau}_{0}$ and $\hat{\sigma}_{0}$. All of the functions satisfy the symmetry relation $\hat{K}\left(x, \boldsymbol{k}, i \omega_{n}\right)=$ $\left[\hat{\sim}\left(x,-\boldsymbol{k}, i \omega_{n}\right)\right]^{*}$, where the unit vector $\boldsymbol{k}$ represents the direction of the Fermi momentum.

The Eilenberger equation (1) is supplemented by a (nonlinear) normalization condition $\breve{g}^{2}=\breve{1}$. It is implemented explicitly by the so-called Riccati parametrization [74-78]. The Green function is expressed in terms of the coherence function $\hat{\gamma}$ in the following way $[77,78]$ :

$$
\begin{gathered}
\check{g}=2\left(\begin{array}{rr}
\hat{\mathcal{G}} & \hat{\mathcal{F}} \\
-\hat{\mathcal{F}} & -\hat{\mathcal{G}}
\end{array}\right)-\check{\tau}_{3}, \\
\hat{\mathcal{G}}=(1-\hat{\gamma} \underset{\sim}{\hat{\gamma}})^{-1}, \quad \hat{\mathcal{F}}=(1-\underset{\gamma}{\hat{\gamma}} \underset{\sim}{\hat{\gamma}})^{-1} \hat{\gamma}, \\
\underset{\sim}{\hat{\mathcal{\gamma}}}=(1-\underset{\sim}{\hat{\gamma}} \hat{\gamma})^{-1}, \quad \hat{\sim} \hat{\mathcal{F}}=(1-\hat{\sim} \hat{\gamma})^{-1} \hat{\sim},
\end{gathered}
$$

where $\hat{\gamma}=\hat{\gamma}\left(x, \boldsymbol{k}, i \omega_{n}\right)$ [79]. The Riccati parametrization reduces the Eilenberger equation (1) into the Riccati-type differential equation [75]:

$$
i v_{F} \cdot \nabla \hat{\gamma}+2 i \omega_{n} \hat{\gamma}+\hat{\Delta}-\hat{\gamma} \hat{\sim} \hat{\gamma}=0 .
$$

This Riccati-Eilenberger equation can be simplified for coherence functions incoming from the bulk to

$$
\boldsymbol{v}_{F} \cdot \nabla \gamma+2 \omega_{n} \gamma-\Delta_{k}+\Delta_{k}^{*} \gamma^{2}=0,
$$

where we assume the superconducting order parameter has only one spin component [i.e., $\hat{\Delta}=\Delta_{k}\left(\hat{\sigma}_{\nu} \hat{\sigma}_{2}\right)$ with $v \in$ $\{0,1,2,3\}]$. In this case, the spin structure of the incoming coherence function is $\hat{\gamma}=\gamma\left(i \hat{\sigma}_{v} \hat{\sigma}_{2}\right)$ (see Appendix A for the details). In a homogeneous superconductor, the coherence function is given by

$$
\bar{\gamma}\left(\boldsymbol{k}, i \omega_{n}\right)=\frac{s_{\omega} \Delta_{k}}{\left|\omega_{n}\right|+\sqrt{\omega_{n}^{2}+\left|\Delta_{k}\right|^{2}}},
$$

where the overbar symbol ${ }^{-}$denotes the bulk value, $\bar{\gamma}$ needs to satisfy the condition $\lim _{\omega_{n} \rightarrow \infty} \gamma=0$, and $s_{\omega}=\operatorname{sgn}\left[\omega_{\mathrm{n}}\right]$.

In USCs, the pair potential depends on the direction of the momentum. For isotropic Fermi surfaces, the momentum dependence of the pair potential is given by

$$
\Delta_{k}=\Delta_{\varphi}= \begin{cases}\Delta_{0} & \text { for } s \text { wave, } \\ \Delta_{0} \cos \varphi & \text { for } p_{x} \text { wave, } \\ \Delta_{0} \sin \varphi & \text { for } p_{y} \text { wave, } \\ \Delta_{0} \cos (2 \varphi) & \text { for } d_{x^{2}-y^{2}} \text { wave, } \\ \Delta_{0} \sin (2 \varphi) & \text { for } d_{x y} \text { wave, }\end{cases}
$$

where $\Delta_{0}$ is the amplitude of the pair potential and $\varphi$ characterizes the direction of the Fermi momentum; $k_{x}=\cos \varphi$ and $k_{y}=\sin \varphi$. Note that the interface is perpendicular to the $k_{x}$ direction. The temperature dependence of the pair potential is determined by the self-consistency condition for a homogeneous SC:

$$
\Delta_{0}(T)=2 N_{0} \lambda \frac{\pi}{\beta} \sum_{\omega_{n}>0}^{\omega_{c}} \int \frac{\Delta_{\varphi} \Lambda_{\varphi}}{\sqrt{\omega_{n}^{2}+\Delta_{\varphi}^{2}}} \frac{d \varphi}{2 \pi},
$$


where $N_{0}$ is the density of states (DOS) per spin at the Fermi level $\beta=1 / T, \omega_{c}$ is the BCS cutoff energy, and

$$
\Lambda_{\varphi}= \begin{cases}1 & \text { for } s \text { wave, } \\ 2 \cos \varphi & \text { for } p_{x} \text { wave, } \\ 2 \sin \varphi & \text { for } p_{y} \text { wave, } \\ 2 \cos (2 \varphi) & \text { for } d_{x^{2}-y^{2}} \text { wave } \\ 2 \sin (2 \varphi) & \text { for } d_{x y} \text { wave }\end{cases}
$$

The coupling constant $\lambda$ is given by

$$
\lambda=\frac{1}{N_{0}}\left[\ln \left(\frac{T}{T_{c}}\right)+\sum_{n=0}^{n_{c}} \frac{1}{n+1 / 2}\right]^{-1},
$$

with $n_{c}=\omega_{c} / 2 \pi T$.

In this paper, the temperature dependence of the pair potential is taken into account, however the approximation of a spatially homogeneous pair potential is made.

Spin-dependent interfaces induce a magnetization in the SC. The induced magnetization, density of the magnetic moment, is given by

$$
\begin{gathered}
M(x)=\mu_{B}\left(n_{\uparrow}-n_{\downarrow}\right), \\
n_{\alpha}(x)=\left\langle\Psi_{\alpha}^{\dagger}(x) \Psi_{\alpha}(x)\right\rangle,
\end{gathered}
$$

where $\mu_{B}$ is the effective Bohr magneton, $\alpha=\uparrow$ or $\downarrow$ is the spin index, $\Psi_{\alpha}\left(\Psi_{\alpha}^{\dagger}\right)$ is the annihilation (creation) operator of a quasiparticle with the spin $\alpha$, and $n_{\alpha}$ is the density of $\alpha$-spin quasiparticles. This magnetization can be obtained from the diagonal parts of the quasiclassical Green's function:

$$
\begin{aligned}
M(x) & =\frac{\mu_{B} N_{0} \pi}{i \beta} \sum_{\omega_{n}} \int \operatorname{Tr}\left[\hat{\sigma}_{3} \hat{g}\left(x, \varphi, i \omega_{n}\right)\right] \frac{d \varphi}{2 \pi}, \\
& =2 \pi \mu_{B} N_{0} T \sum_{n=0}^{n_{c}} \int \operatorname{Im}\left[g_{\uparrow}-g_{\downarrow}\right] \frac{d \varphi}{2 \pi},
\end{aligned}
$$

where we have used the symmetry of the Matsubara Green function $\hat{g}\left(x, \varphi, i \omega_{n}\right)=-\hat{g}^{*}\left(x, \varphi,-i \omega_{n}\right)$, and the abbreviation $\hat{g}=\operatorname{diag}\left[g_{\uparrow}, g_{\downarrow}\right]$ with $g_{\uparrow(\downarrow)}$ being the normal Green's function for the up and down spin.

\section{B. Boundary condition}

The boundary conditions for the coherence functions are given in Refs. [76-78]. Hereafter, the outgoing (incoming) coherence functions are denoted by $\Gamma(\gamma)$ as introduced in Ref. [76]. When the SC and F are semi-infinitely long in the $x$ direction, the boundary condition is simplified because $\gamma=0$ in the $\mathrm{F}$. The boundary condition is given by

$$
\hat{\Gamma}=\hat{r} \hat{\gamma} \hat{r}^{*}
$$

where the reflection-coefficient matrix $\hat{r}$ is given by [80]

$$
\hat{r}=\left[\begin{array}{cc}
r_{\uparrow} & 0 \\
0 & r_{\downarrow}
\end{array}\right]=\left[\begin{array}{cc}
\left|r_{\uparrow}\right| e^{i\left(\phi+\theta_{\mathrm{SM}}\right)} & 0 \\
0 & \left|r_{\downarrow}\right| e^{i\left(\phi-\theta_{\mathrm{SM}}\right)}
\end{array}\right] .
$$

The angle $\theta_{\mathrm{SM}}$ is the so-called spin-mixing angle [64] and $r_{\uparrow}$ and $r_{\downarrow}$ are the reflection coefficients for the up-spin and downspin particles injected from the $\mathrm{SC}$ side. The physical meaning of $\theta_{\mathrm{SM}}$ is explained in Appendix E. The reflection coefficients (a) $g\left(x_{0},+k, \varepsilon\right)$

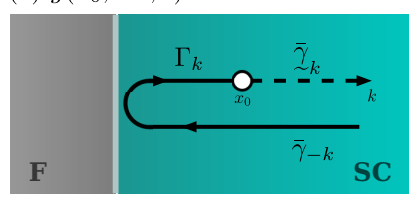

(b) $g\left(x_{0},-k, \varepsilon\right)$

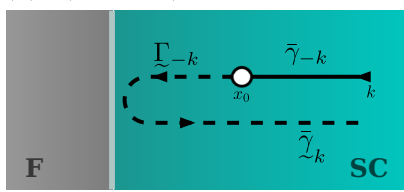

FIG. 2. Quasiclassical path to obtain the Green's function in $\left(+k, k_{y}\right)$ and $\left(-k, k_{y}\right)$ direction, respectively. The particle-like (holelike) coherence function must be solved along the quasiclassical path in $+k(-k)$ direction, which are indicated by the solid and broken lines. The points of interest are denoted by $x_{0}$. The momentum parallel to the interface is conserved during the reflection. The overbar symbol ${ }^{-}$denotes the bulk value of the coherence function.

can be obtained by matching the wave functions:

$$
r_{\alpha}=\frac{\hbar\left(v-v_{\alpha}\right)-2 i V}{\hbar\left(v+v_{\alpha}\right)+2 i V},
$$

where we assume a potential barrier $V \delta(x)$ at the interface, and $v=\hbar k / m^{*}\left(v_{\alpha}=\hbar k_{\alpha} / m^{*}\right)$ is the $x$ component of the Fermi velocity $v_{F}$ in the SC $(\mathrm{F})$ side with $m^{*}$ being the quasiparticle effective mass. We also introduce a dimensionless barrier potential parameter $z_{0}=V /\left(\hbar v_{F}\right)$.

The reflection coefficients in the boundary condition are obtained by matching the wave functions at the interface. The wave functions are obtained from the single-particle Hamiltonian, which is given by

$$
\hat{\mathcal{H}}= \begin{cases}-\frac{\hbar^{2}}{2 m^{*}}\left(\partial_{x}^{2}+\partial_{y}^{2}\right)-E_{F}-J_{\mathrm{ex}} \hat{\sigma}_{3} & \text { for } \quad x<0, \\ -\frac{\hbar^{2}}{2 m^{*}}\left(\partial_{x}^{2}+\partial_{y}^{2}\right)-E_{S} & \text { for } x \geqslant 0,\end{cases}
$$

where $J_{\mathrm{ex}} \hat{\sigma}_{3}$ is the exchange energy in the $\mathrm{F}$ and $E_{F(S)}$ are related to the Fermi energies in the $\mathrm{F}(\mathrm{S})$ region (measured from the bottom of the energy bands, respectively; the electrochemical potential defines zero energy). Therefore, the wave numbers at the Fermi level are given by $k=\left\{2 m^{*} E_{S} / \hbar^{2}-\right.$ $\left.k_{y}^{2}\right\}^{1 / 2}$, and $k_{\uparrow(\downarrow)}=\left\{2 m^{*}\left[E_{F}+(-) J_{\text {ex }}\right] / \hbar^{2}-k_{y}^{2}\right\}^{1 / 2}$. Note that we have made $\hbar$ explicit for convenience.

In a single-spin-component superconductor, the coherence amplitude propagating from the bulk region can be expressed as

$$
\hat{\bar{\gamma}}=\bar{\gamma}\left(i \hat{\sigma}_{\nu} \hat{\sigma}_{2}\right),
$$

Therefore, the outgoing coherence functions (16) are given by

$$
\hat{\Gamma}=\left(\begin{array}{cc}
0 & \Gamma_{\uparrow} \\
s_{\nu} \Gamma_{\downarrow} & 0
\end{array}\right)=\left(\begin{array}{cc}
0 & r_{\uparrow} \gamma r_{\downarrow}^{*} \\
s_{\nu} r_{\downarrow} \gamma r_{\uparrow}^{*} & 0
\end{array}\right),
$$

for the opposite-spin pairing ( $v=0$ or 3 ), where $s_{v}=1(-1)$ for the spin-triplet (singlet) pairing. The boundary conditions obtained here are consistent with those derived using the socalled evolution operators [81-84].

To obtain the coherence amplitude, we need to consider the group velocity of the quasiparticle and quasihole properly. The quasiclassical paths to obtain $\breve{g}\left(x_{0}, k, k_{y}, i \omega_{n}\right)$ and $\breve{g}\left(x_{0},-k, k_{y}, i \omega_{n}\right)$ are shown in Figs. 2(a) and 2(b), where the solid and broken lines represent the path for the particle-like and hole-like coherence amplitudes, the arrows indicate the direction of the Fermi momentum, and $k_{y}$ is the momentum parallel to the interface. Since the quasiparticle (quasihole) 


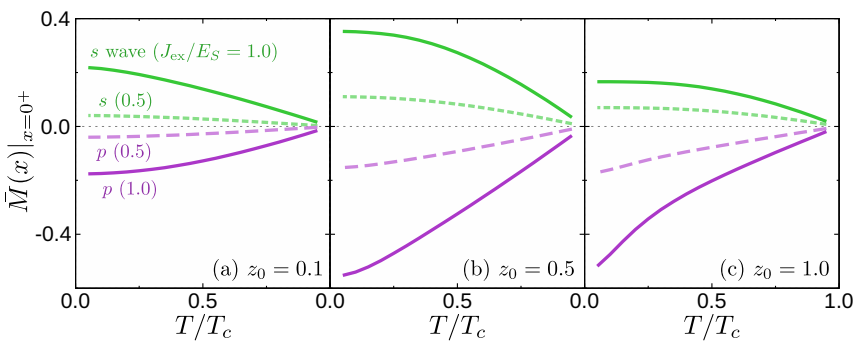

FIG. 3. Temperature dependence of induced magnetization at the interface of a $1 D \mathrm{FM} / \mathrm{SC}$ model. The induced magnetization is normalized as $\bar{M}=M / 2 \pi \mu_{B} N_{0} T_{c}$. The exchange energy is set to $J_{\text {ex }}=E_{F}$ or $0.5 E_{F}$ with $E_{F}=E_{S}$. The spin-independent barrier potential is set to (a) $z_{0}=0.1$, (b) 0.5 , and (c) 1.0 , where $\theta_{\mathrm{SM}}<0$ for all of the sets of the parameters. The induced magnetization of the $s$-wave junctions is positive, whereas that of the $p$-wave junctions are negative. The pair potential depends on the temperature but is kept constant as function of the spatial coordinate $x$.

propagates in the same (opposite) directions as $\boldsymbol{k}, \hat{\gamma}$ and $\hat{\gamma}$ should be solved in $\boldsymbol{k}$ and $\boldsymbol{- k}$ directions, respectively.

The Green's function can be obtained from the coherence functions [see Eq. (3)]. Using the boundary condition, the diagonal part of the Green's functions at the interface are given in terms of the coherence functions:

$$
\begin{aligned}
& \hat{g}_{+k}=(1-\hat{\Gamma} \underset{\sim}{\hat{\gamma}})^{-1}(1+\hat{\Gamma} \underset{\sim}{\hat{\gamma}}), \\
& \hat{g}_{-k}=(1-\hat{\gamma} \underset{\sim}{\hat{\Gamma}})^{-1}(1+\hat{\gamma} \underset{\sim}{\hat{\Gamma}}),
\end{aligned}
$$

where $\hat{g}_{ \pm k}=\hat{g}\left(x=0^{+}, \pm k, k_{y}, i \omega_{n}\right)$. The spin-reduced Green's functions at the interface are

$$
g_{\alpha,+k}=\frac{1+\Gamma_{\alpha} \underline{\gamma}}{1-\Gamma_{\alpha} \underline{\gamma}}, \quad g_{\alpha,-k}=\frac{1+\gamma \underline{\Gamma}_{\alpha^{\prime}}}{1-\gamma \underline{\Gamma}_{\alpha^{\prime}}},
$$

where $\alpha^{\prime}$ means the opposite spin of $\alpha$. The spin structure of the coherence functions are parameterized as $\hat{\Gamma}=\operatorname{diag}\left[\Gamma_{\uparrow}, \Gamma_{\downarrow}\right]\left(i \hat{\sigma}_{\nu} \hat{\sigma}_{2}\right)$ and $\hat{\Gamma}=\operatorname{diag}\left[\Gamma_{\uparrow}, \Gamma_{\downarrow}\right]\left(i \hat{\sigma}_{\nu} \hat{\sigma}_{2}\right)^{*}=$ $\operatorname{diag}\left[\underline{\Gamma}_{\uparrow}, \underline{\Gamma}_{\downarrow}\right]\left(i \hat{\sigma}_{\nu} \hat{\sigma}_{2}\right)^{\dagger}$. Assuming the spatially homogeneous pair potential, we can replace $\gamma$ in Eq. (24) by $\bar{\gamma}$, where the symbol - means bulk values. This assumption changes the results only quantitatively but not qualitatively.

\section{ONE-DIMENSIONAL MODEL}

In order to understand the basics of the IPE. We start with one-dimensional (1D) models (i.e., superconducting wire). Such systems can be considered by setting $k_{y}=0$.

\section{A. Ferromagnetic-metal junction}

The temperature dependence of the induced magnetizations at the interface of the F/SC junction are shown in Fig. 3 where spin-singlet even-parity and spin-triplet odd-parity superconducting junctions are considered, which correspond to the $s$ - and $p_{x}$-wave superconducting junctions in the 2D case, respectively. The exchange energy in the $\mathrm{F}$ is set to $J_{\text {ex }}=E_{F}$ or $0.5 E_{F}$ with $E_{F}=E_{S}$, the barrier potential is set to (a) $z_{0}=0.1$, (b) $z_{0}=0.5$, and (c) $z_{0}=1.0$, and the pair potential is assumed spatially homogeneous but temperature dependent. In the even-parity case, the magnetization in the

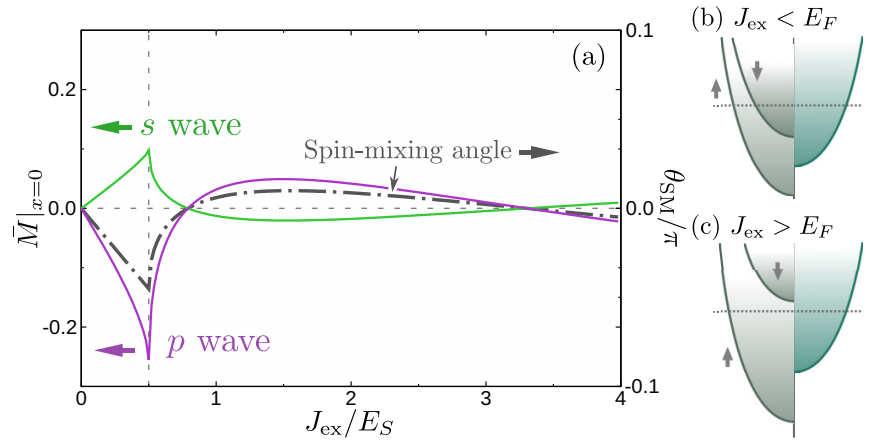

FIG. 4. (a) Induced magnetization and spin-mixing angle of a $1 D$ $\mathrm{F} / \mathrm{SC}$ system as a function of $J_{\text {ex }}$. The Fermi energy in the $\mathrm{F}$ is set to $E_{F}=0.5 E_{S}$, which means that the $\mathrm{F}$ is a FM (HM) when $J_{\text {ex }}<0.5 E_{S}$ $\left(J_{\mathrm{ex}} \geqslant 0.5 E_{S}\right)$. The schematic band structures of the FM/SC and $\mathrm{HM} / \mathrm{SC}$ junctions are shown in (b) and (c) respectively. The up-spin and down-spin subbands for the $\mathrm{F}$ are shown in the left side, whereas the band for the SC is in the right side. The dotted line indicates the Fermi level. The sign of the magnetization is determined by $\operatorname{sgn}\left(J_{\mathrm{ex}}\right)$ and the pairing symmetry. The temperature and the barrier potentials are set to $T=0.2 T_{c}$ and $z_{0}=1$.

F induces the parallel magnetization in the SC as shown in Fig. 3. This behavior is consistent with that in the ballistic limit in Refs. [66,67]. In the odd-parity case, on the contrary, the induced magnetization is antiparallel to the magnetization in the F. We have confirmed that no magnetization is induced when the $d$ vector is perpendicular to the magnetization vector in the $\mathrm{F}$.

The induced magnetization $\left.M\right|_{x=0^{+}}$and the spin-mixing angle $\theta_{\mathrm{SM}}$ as functions of $J_{\mathrm{ex}}$ are shown in Fig. 4(a) where $E_{F}=0.5 E_{S}, z_{0}=1.0$, and $T=0.2 T_{c}$ [85]. In this case, the $\mathrm{F}$ is a ferromagnetic metal (FM) for $0<J_{\mathrm{ex}}<E_{F}$ and a half-metal (HM) for $J_{\mathrm{ex}}>E_{F}$ as schematically illustrated in Figs. 4(b) and 4(c). As shown in Fig. 4(a), the sign of $\left.M\right|_{x=0^{+}}$for the $s$-wave junction is always opposite to $\operatorname{sgn}\left[\theta_{\mathrm{SM}}\right]$, whereas that for the $p$-wave junction always has the same sign. These results demonstrate that the sign of the induced magnetization is determined by the two factors: the pairing symmetry and the sign of the spin-mixing angle $\theta_{\mathrm{SM}}$.

\section{B. Ferromagnetic-insulator junction}

The IPE occurs in ferromagnetic-insulator(FI)/SC junctions as well. The $J_{\text {ex }}$ dependence of $\left.M\right|_{x=0^{+}}$is shown in Fig. 5(a). In order to model the FI, we set $E_{F}=-E_{S}$ where the FI-HM transition occurs at $J_{\mathrm{ex}}=\left|E_{F}\right|$ as schematically shown in Figs. 5(b) and 5(c). Figure 5(a) shows that $\left.M\right|_{x=0^{+}}$in the $s$-wave junction is antiparallel to the magnetization in the F. This result is consistent with Ref. [64] (see [86]). For the $p$-wave case, on the other hand, the induced magnetization is parallel to the magnetization of the $\mathrm{F}$. We can conclude that the IPE induces the magnetization with the opposite sign compared with an $s$-wave junction.

At low temperature, $|M|$ in $J_{\mathrm{ex}}<E_{F}$ for the $p$-wave junction is greatly larger than that for the $s$-wave case. Similar low-temperature anomalies of the magnetic response have been reported so far [43-45,45,47-49,52,53]. These anomalies are explained by the emergence of the zero-energy 


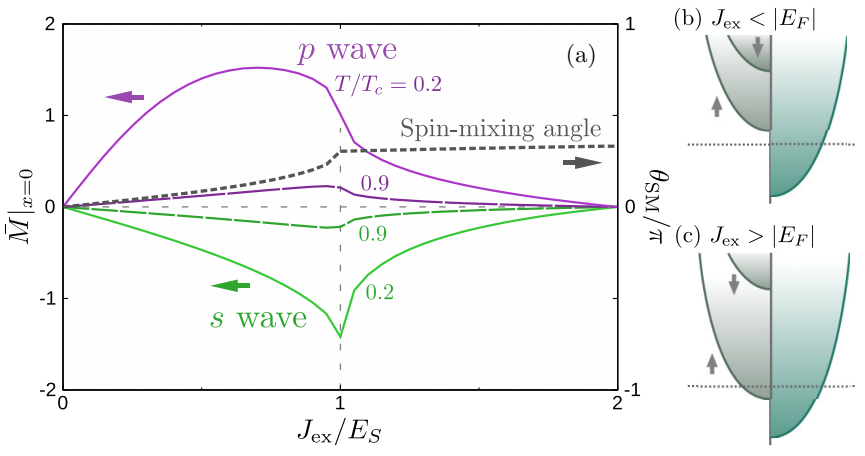

FIG. 5. (a) Induced magnetization and spin-mixing angle of a $1 D$ $\mathrm{F} / \mathrm{SC}$ model with $E_{F}=-E_{S}$. The $\mathrm{F}$ is insulating for $J_{\mathrm{ex}}<E_{S}$ or a half-metallic for $J_{\mathrm{ex}}>E_{S}$. The induced magnetization for an $s$-wave junction is negative regardless of the temperature (i.e., $T=0.2 T_{c}$ and $0.9 T_{c}$ ), whereas that $p$-wave junctions are positive. The barrier potential is set to $z_{0}=0$. The $s$-wave results are consistent with those in Ref. [64], where $\theta_{\mathrm{SM}}$ is defined with the opposite sign compared with our definition. The schematics of band structures of the FI/SC and $\mathrm{HM} / \mathrm{SC}$ junctions are shown in (b) and (c) in the same manner as in Figs. 4(b) and 4(c).

ABSs. In superconducting junctions, ABSs appear when $\operatorname{sgn}\left[\Delta\left(k_{x}, k_{y}\right) \Delta\left(-k_{x}, k_{y}\right)\right]=-1$ because of the interference between the quasiparticle propagating into an interface and reflected one. Therefore, the effects of ABSs become larger as increasing reflection probability $|R|[6]$. In other words, the anomalous IPE becomes more prominent in an FI/SC junction than that in FM/SC and HM/SC junctions. The relation between the ABSs and the direction of the induced magnetization is discussed in Appendix C. Using the magnetic-wall model, we analytically derive the magnetization in an SC and demonstrate that an ABS inverses the induced magnetization.

The amplitude of $\left.M\right|_{x=0^{+}}$changes suddenly at $J_{\mathrm{ex}}=\left|E_{F}\right|$ in accordance with the FI-HM transition. After the FI-HM transition, the induced magnetization decreases with increasing $J_{\mathrm{ex}}$ and vanishes at $J_{\mathrm{ex}}=2\left|E_{F}\right|$ regardless of the pairing symmetry. When $E_{F} \pm J_{\mathrm{ex}}=E_{S}$, the dispersion relation of either band in the $\mathrm{F}$ becomes identical to that in the $\mathrm{SC}$, with the consequence that the reflection probability for the corresponding spin becomes zero. In this case, both $\Gamma_{\uparrow}$ and $\Gamma_{\downarrow}$ are zero [see Eq. (21)], which means the IPE does not occur.

\section{Induced magnetization and pair amplitudes}

The induced magnetization can be expressed in terms of the pair amplitude (i.e., anomalous Green's function) when $T \sim T_{c}$ (see Appendix D for the details) [52,87]. In the 1D limit, in particular, the magnetization is given by

$$
\begin{gathered}
M \approx 4 \pi \mu_{B} N_{0} T \sum_{\omega_{n}>0} m_{0,3}, \\
m_{\nu, v^{\prime}}=\operatorname{Im}\left[f_{\nu, \mathrm{SW}} f_{v^{\prime}, \mathrm{SW}}^{*}+f_{\nu, \mathrm{PW}} f_{\nu^{\prime}, \mathrm{PW}}^{*}\right],
\end{gathered}
$$

where SW and PW stand for the $s$-wave and $p$-wave pairings, respectively. The spin indices $v=0$ and 3 represent the spin-singlet and spin-triplet pairs respectively. Note that $f_{0, \mathrm{PW}}$ and $f_{3, \mathrm{SW}}$ should be odd-functions of $\omega_{n}$ to satisfy the Pauli rule $[10,22,88]$. Equation (25) means that the magnetization is given by the product of the spin-singlet and spin-triplet pairs.
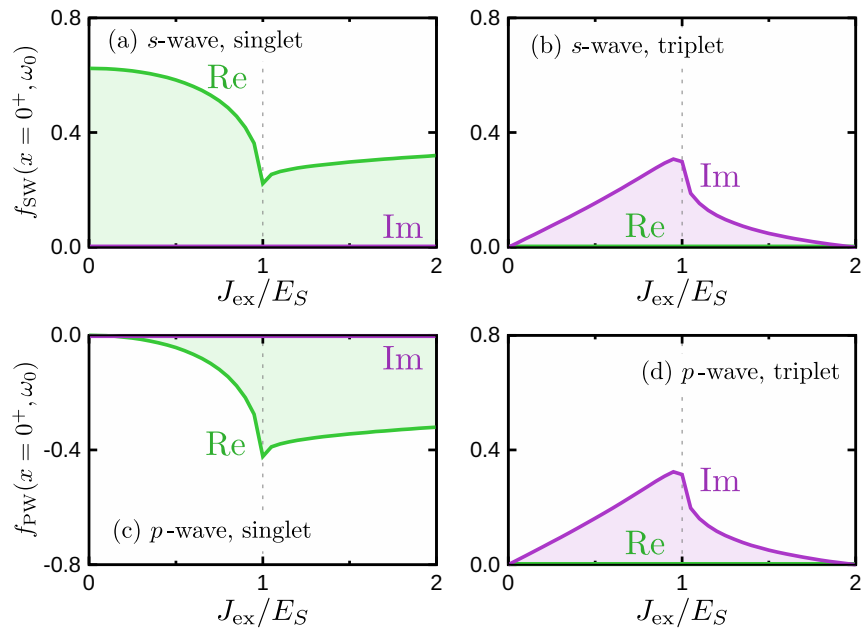

FIG. 6. Pair amplitudes in a $1 D \mathrm{~F} / s$-wave model. The $s$-wave spin-singlet, $s$-wave triplet, $p$-wave singlet, and $p$-wave triplet components are plotted in (a), (b), (c), and (d). When $J_{\text {ex }}<E_{S}$ (i.e., FI regime), the magnetization is mainly generated by the product of the $s$-wave singlet and $s$-wave triplet pairs because the $s$-wave singlet is dominant [see Eq. (25)]. The temperature and the Matsubara frequency are set to $T=0.9 T_{c}$ and $\omega_{n}=\omega_{0}$.

The pair amplitudes at the interface of the $s$-wave junction (i.e., junction with an $s$-wave SC) are shown in Fig. 6, where $T=0.9 T_{c}$ and $\omega=\omega_{0}=\pi T$. The spin-singlet $s$-wave, spintriplet $s$-wave, spin-singlet $p$-wave, and spin-triplet $p$-wave pair amplitudes are shown in Figs. 6(a), 6(b), 6(c), and 6(d). In the FI region (i.e., $J_{\mathrm{ex}}<E_{F}$ ) of an $s$-wave junction, the conventional spin-singlet $s$-wave pairs are dominant and the other pair amplitudes are relatively small. The magnetization in this case is mainly generated by the spin-singlet and spintriplet $s$-wave Cooper pairs (i.e., $f_{0, \mathrm{Sw}} f_{3, \mathrm{Sw}}^{*} \gg f_{0, \mathrm{PW}} f_{3, \mathrm{PW}}^{*}$ when $J_{\mathrm{ex}} \ll E_{F}$ ).

In the $p$-wave case, on the other hand, the spin-triplet $s$ wave pair amplitude is dominant for $J_{\mathrm{ex}}<E_{F}$ as shown in Fig. 7. In addition, the spin-triplet $s$-wave pair amplitudes
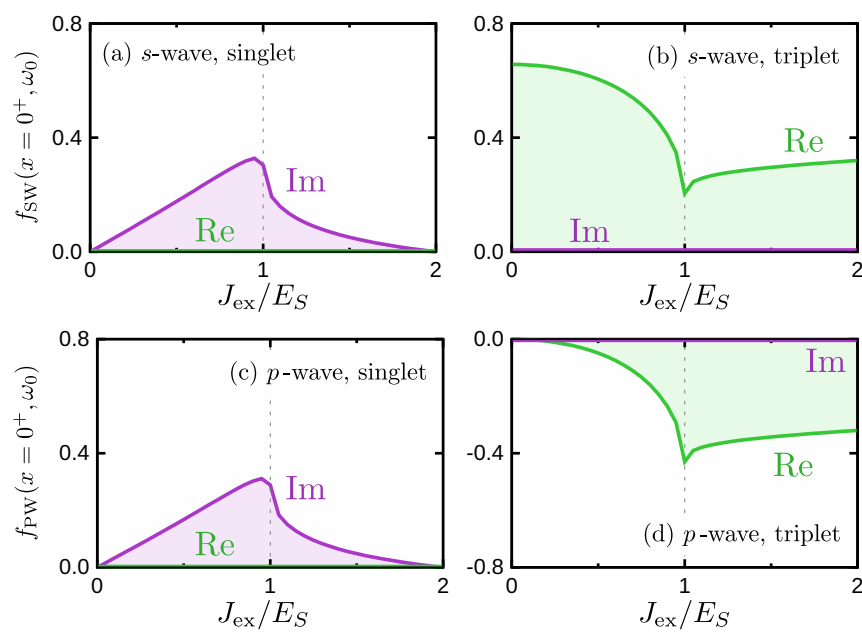

FIG. 7. Pair amplitudes in a $1 \mathrm{D} \mathrm{F} / p$-wave model. The results are plotted in the same manner as in Fig. 6. The main contribution comes from the $s$-wave pairs even in the $p$-wave spin-triplet superconducting junction. 

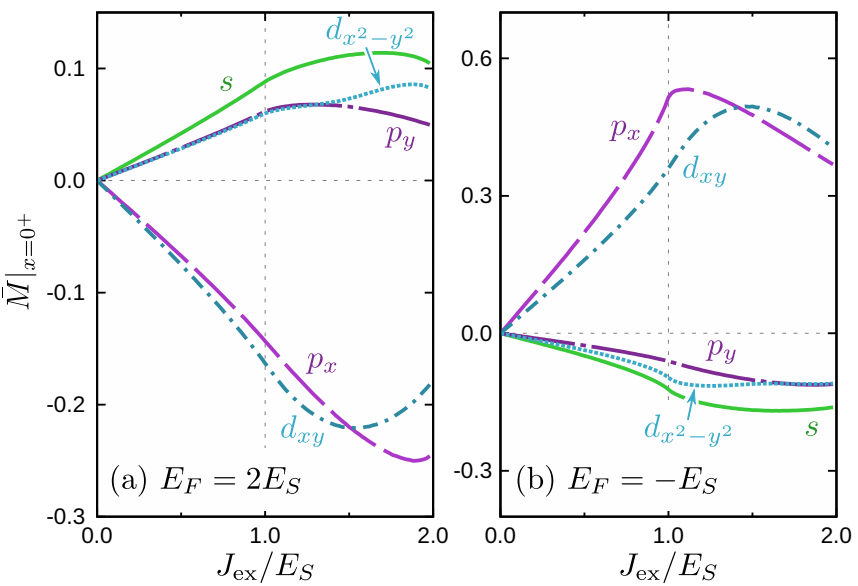

FIG. 8. Induced magnetization of a $2 D \mathrm{~F} / \mathrm{SC}$ junction. The Fermi energy is set to (a) $E_{F}=2 E_{S}$ and (b) $E_{F}=-E_{S}$. When the ABSs are present, the induced magnetizations have the opposite sign to those without ABSs. The temperature and the barrier potentials are set to $T=0.2 T_{c}$ and $z_{0}=1$.

have almost the same $J_{\text {ex }}$ dependencies for the spin-singlet $s$-wave pair amplitude as shown in Figs. 6(a) and 7(b). Comparing Figs. 6 and 7, similar correspondences between spin-singlet and spin-triplet pairs are confirmed. In Eqs. (25) and (26), such a singlet-triplet conversion results in the sign change of the magnetization (i.e., $m_{0,3}=-m_{3,0}$ ). Namely, the $s$-wave pairs in the $p$-wave junction generate almost the same amplitude of the magnetization compared with the one in the $s$-wave junction. The direction, however, is opposite compared with that in the $s$-wave junction. In the Cooper pair picture, the spin structure of the dominant Cooper pair determines the direction of the induced magnetization.

\section{TWO-DIMENSIONAL MODEL}

Most realistic superconducting junctions are twodimensional or three-dimensional. In a $2 \mathrm{D}$ system (i.e., junction with a two-dimensional SC), local quantities should be obtained via a $k_{y}$ integration, where $k_{y}$ is the momentum parallel to the interface [see Eq. (15)]. In particular, the induced magnetization generated by the IPE is obtained via $k_{y}$ integration where the partial magnetization depends on $k_{y}$ via the transport coefficients and the pair potential.

The induced magnetizations in the 2D junctions are shown in Fig. 8, where the Fermi energy in the $\mathrm{F}$ is set to (a) $E_{F}=2 E_{S}$ and (b) $E_{F}=-E_{S}$. When $E_{F}=2 E_{S}, \operatorname{sgn}(M)$ is determined by whether the ABSs are present or not. The anomalous IPE occurs when the ABSs are present at the interface (i.e., $p_{x}$ - and $d_{x y}$-wave junctions). When $J_{\mathrm{ex}}<E_{F}$, all of the channels are regarded as FM/SC junctions, which results in the parallel (antiparallel) magnetization in the SC without (with) ABSs as discussed in the 1D limit [see Fig. 4(a)]. The direction of the induced magnetization does not change even in the $J_{\text {ex }}>E_{S}$ region.

When $E_{F}=-E_{S}, \operatorname{sgn}(M)$ for each superconducting junction is inverted compared with Fig. 8(a). In the $J_{\mathrm{ex}}<E_{F}$

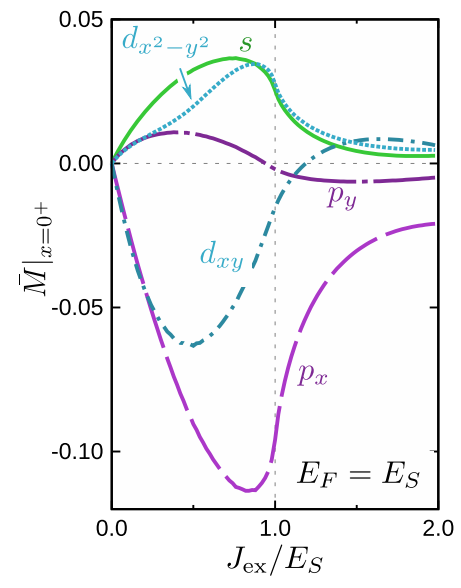

FIG. 9. Induced magnetization of a $2 \mathrm{D} \mathrm{F} / \mathrm{S}$ junction with $E_{F}=$ $E_{S}$. The results are plotted in the same manner in Fig. 8. When $J_{\text {ex }}$ is sufficiently large, the induced magnetizations for an $s$-wave and $p_{y}$-wave junctions are opposite even though no ABS appears in both of the junctions.

region, all of the channels are regarded as an $\mathrm{FI} / \mathrm{SC}$ junction where the induced magnetization in the absence (presence) of ABSs is negative (positive) as discussed in the 1D limit [see Fig. 5(a)]. When $J_{\text {ex }}>E_{F}$, even though the channels around $k_{y}=0$ change to $\mathrm{HM} / \mathrm{SC}$ junctions, the sign of $\theta_{\mathrm{SM}}$ remains unchanged. Therefore, the total amplitude of the magnetization also remains unchanged.

When $J_{\mathrm{ex}} \gg E_{F}$ and $E_{F}=E_{S}$, the sign change of $\theta_{\mathrm{SM}}$ can not be ignored. The $J_{\mathrm{ex}}$ dependence of $\left.M\right|_{x=0^{+}}$for $E_{F}=E_{S}$ are shown in Fig. 9. In the $p_{y^{-}}$and $d_{x y}$-wave case (i.e., SCs with gap nodes at $k_{y}=0$ ), the direction of the induced magnetization changes around $J_{\mathrm{ex}}=E_{F}$ as shown in Fig. 9. On the other hand, the signs of $\left.M\right|_{x=0^{+}}$for the $s^{-}, p_{x^{-}}$, and $d_{x^{2}-y^{2}}$ wave superconducting junction (i.e., SCs without gap does at $\left.k_{y}=0\right)$ are unchanged.

To understand the sign change of the induced magnetization, we evaluated the angle-resolved magnetization $M(\varphi)$ with $k_{y}=\sin \varphi$. The results for $s$ - and $p_{y}$-wave junctions with $E_{F}=2 E_{S}$ are shown in Figs. 10(a) and 10(b). In this case, $M(\varphi)$ are positive for both of the junctions because the sign of $\theta_{\mathrm{SM}}$ is always positive as shown in Fig. 10(c). Note that $\left.M(\varphi)\right|_{\varphi=0}=0$ in the $p_{y}$-wave junction because of the nodes on the superconducting gap shown by the broken red line in Fig. 10(b).

The results with $E_{F}=E_{S}$ are shown in Figs. 10(d), 10(e), and 10(f). The spin-mixing angle can be negative when $J_{\mathrm{ex}} \neq$ 0 as shown in Fig. 10(f) [39]. In the $s$-wave junction with $J_{\mathrm{ex}} \neq 0$, the positive contribution (shown in green) around $\varphi=0$ is larger than the negative ones (shown in purple) around $|\varphi|=\pi / 2$. The total magnetization, therefore, is always positive even for $E_{F}=E_{S}$ as shown in Fig. 9. In the $p_{y}$-wave junction, on the other hand, the positive contribution is smaller than in the $s$-wave case because of the nodes. As a result, the direction of the total magnetization can change around $J_{\mathrm{ex}}=E_{F}$ (Fig. 9). 

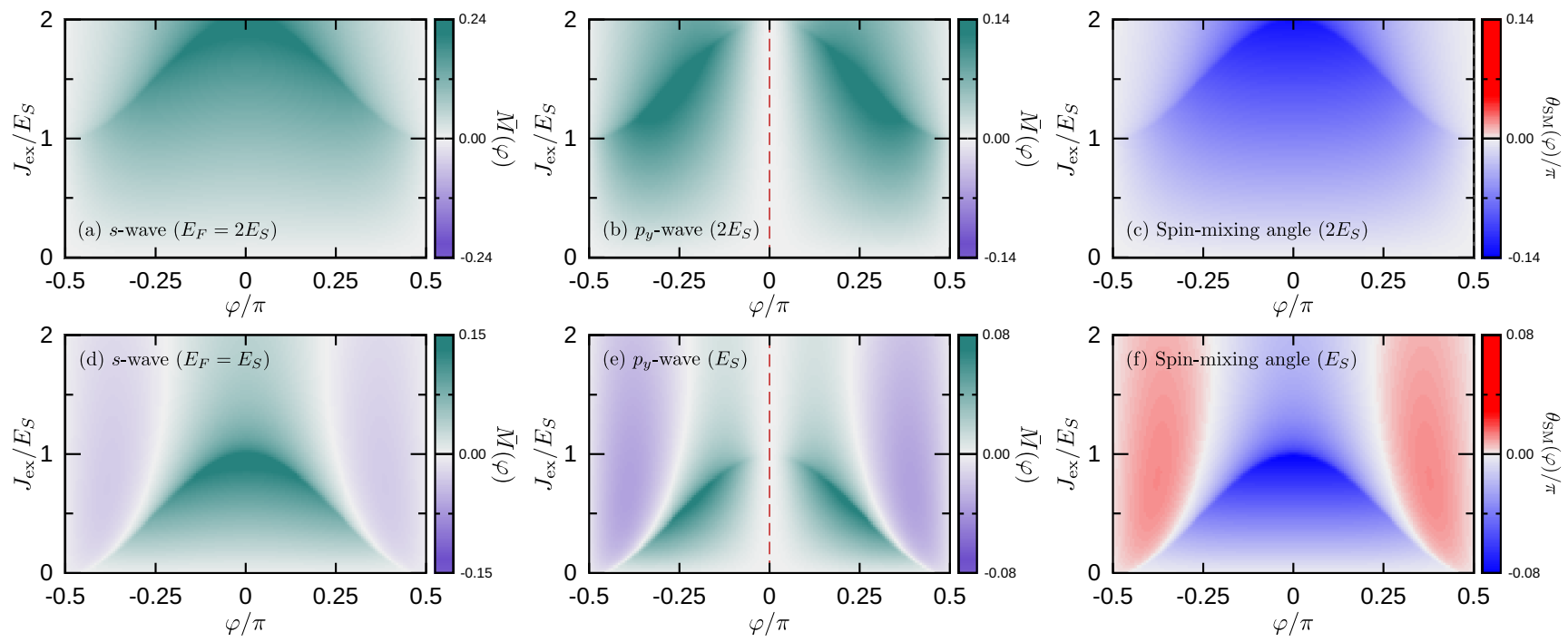

FIG. 10. Angle-resolved magnetization and spin-mixing angle of $\mathrm{F} / \mathrm{SC}$ junctions. The Fermi energy in the $\mathrm{F}$ is set to $E_{F}=2 E_{S}$ in (a)-(c), whereas $E_{F}=E_{S}$ in (d)-(f). The order parameter is assumed spin-singlet $s$-wave in (a) and (d), and spin-triplet $p$-wave in (b) and (e). When $E_{F}=E_{S}$ and $J_{\mathrm{ex}} \neq 0$, the sign change of $\theta_{\mathrm{SM}}$ occurs around $k_{y} \sim \pm k_{F}$. In (e), when $J_{\mathrm{ex}}>E_{F}$, the positive contribution to $M$ is smaller than the negative one due to the nodes of the $p_{y}$-wave gap at $\varphi=0$. In (a), (b), (d), and (e), the partial magnetizations are normalized to $\bar{M}(\varphi)=M(\varphi) / 2 \pi \mu_{B} N_{0} T_{c}$ and the exchange energy in $\mathrm{F}$ is changed from $J_{\mathrm{ex}}=0.8$ to 2.0 by 0.2 . The temperature and the barrier potentials are set to $T=0.2 T_{c}$ and $z_{0}=0$.

\section{DISCUSSIONS}

The relations between the induced magnetization and the magnetization of the $\mathrm{F}$ are summarized in Table I. In the table, the sign of $\theta_{\mathrm{SM}}$ is determined in the weak-magnetization limit (i.e., $J_{\text {ex }} \ll E_{F}$ ). We see that the ABSs always inverse the induced magnetization by the IPE.

The anomalous IPE presented in this paper can be observed by ferromagnetic resonance (FMR) measurements [72,73], by nuclear magnetic resonance (NMR) measurements [68], and by polar Kerr effect measurements [69]. In these experiments the conventional IPE has been observed. Replacing the conventional SC by an USC such as a high- $T_{c}$ cuprate, it would possible to confirm experimentally the anomalous IPE.

In this paper, we assume that the pair potential depends only on the temperature but not on the coordinate. This assumption, however, changes the results only quantitatively

TABLE I. Direction of the induced magnetization. The parallel (antiparallel) arrows mean that the induced magnetization by the IPE is parallel (antiparallel) to the magnetization in the $\mathrm{F}$, respectively. We assume the exchange energy is weak enough (i.e., $J_{\mathrm{ex}} \ll E_{F}$ ). In the table, ABS, FI, and FM stand for Andreev bound state, ferromagnetic insulator, and ferromagnetic metal.

\begin{tabular}{l}
\hline $\mathrm{w} / \mathrm{O} \mathrm{ABS} \quad \mathrm{w} / \mathrm{ABS}$ \\
$\mathrm{FM}\left(\theta_{\mathrm{SM}}>0\right)$
\end{tabular}

but not qualitatively. Our main conclusion about the direction of the induced magnetization would remain unchanged even if we employ the spatial-dependent self-consistent pair potential.

\section{CONCLUSION}

We have theoretically studied the IPE in F/USC junctions utilizing the quasiclassical Green function theory. We have shown that the direction of the induced magnetization is determined by two factors: by whether the ABS exists and by the sign of the spin-mixing angle $\theta_{\mathrm{SM}}$. Namely, in the 1D limit, the induced magnetizations for the $p_{x}$-wave $\mathrm{SC}$ is always opposite to that for the $s$-wave SC.

In the $2 \mathrm{D}$ model, the spin-mixing angle $\theta_{\mathrm{SM}}$ depends on the momentum parallel to the interface $k_{y}$. The results for 2D $\mathrm{F} / \mathrm{SC}$ junctions are qualitatively the same as those in the 1D limit when the exchange energy in the $\mathrm{F}\left(J_{\mathrm{ex}}\right)$ is smaller than the Fermi energy of the $\mathrm{F}\left(E_{F}\right)$. When $J_{\text {ex }} \gg E_{F}$, the sign of the induced magnetization is not simply determined by the ABSs because the sign changes of $\theta_{\mathrm{SM}}$ around $k_{y} \sim \pm k_{F}$ are not negligible in this parameter range.

In addition, analyzing the pair amplitudes in 1D models, we have pointed out a correspondence at $T \sim T_{c}$ between the spin-singlet pairs in an $s$-wave junction and the spin-triplet pairs in a $p$-wave junction. The odd-frequency spin-triplet $s$-wave pairs induced at the interface of the spin-triplet $p$-wave junction have qualitatively the same $J_{\text {ex }}$ dependence as that for the spin-singlet $s$-wave pairs induced in the $s$-wave junction. Reflecting this correspondence, the amplitudes of the induced magnetizations in the $s$ - and $p$-wave SC junctions are qualitatively the same. Their directions, however, are opposite to each other, where the direction of the magnetization is determined by the relative phase between the spin-singlet and spin-singlet pair functions. 


\section{ACKNOWLEDGMENTS}

This work was supported by the JSPS Core-to-Core program "Oxide Superspin" international network, Grantsin-Aid from JSPS for Scientific Research on Innovative Areas "Topological Materials Science" (KAKENHI Grants No. JP15H05851, No. JP15H05852, No. JP15H05853, and No. JP15K21717), Scientific Research (A) (KAKENHI Grant No. JP20H00131), Scientific Research (B) (KAKENHI Grants No. JP18H01176 and No. JP20H01857), JapanRFBR Bilateral Joint Research Projects/Seminars No. 19-5250026. S.-I.S. acknowledges hospitality at the University of Greifswald.

\section{APPENDIX A: SPIN STRUCTURES OF PAIR POTENTIAL}

The index $v$ is a parameter, which decides the spinstructure of the superconductor. The pair potential is generally expressed as a mixture of different spin structures:

$$
\hat{\Delta}=\left[\begin{array}{cc}
\Delta_{\uparrow \uparrow} & \Delta_{\uparrow \downarrow} \\
\Delta_{\downarrow \uparrow} & \Delta_{\downarrow \downarrow}
\end{array}\right]=\sum_{\nu=0}^{3} \Delta_{\nu} \hat{\sigma}_{\nu} \hat{\sigma}_{2},
$$

where $\Delta_{v=0}$ is the spin-singlet component, $\Delta_{v=3}$ is the opposite-spin spin-triplet one, and $\Delta_{\nu=1}$ and $\Delta_{\nu=2}$ are the equal-spin spin-triplet ones. In this paper, for simplicity, we assume the single-spin-component superconductor for which the pair-potential matrix is given by

$$
\hat{\Delta}=\Delta_{\nu} \hat{\sigma}_{\nu} \hat{\sigma}_{2}
$$

with $v$ being $0,1,2$, or 3 . The spin structure of the coherence function $\hat{\gamma}$ incoming from the bulk is parameterized in the same way. Writing $\hat{\Delta} \equiv \hat{\Delta}_{k}$ and $\Delta_{v} \equiv \Delta_{k}$ (we omit the index $v$ for brevity), Eq. (6) is then written as

$$
\begin{gathered}
i \boldsymbol{v}_{F} \cdot \nabla \gamma\left(i \hat{\sigma}_{\nu} \hat{\sigma}_{2}\right)+2 i \omega_{n} \gamma\left(i \hat{\sigma}_{\nu} \hat{\sigma}_{2}\right)+\Delta_{k}\left(\hat{\sigma}_{\nu} \hat{\sigma}_{2}\right) \\
+\gamma\left(i \hat{\sigma}_{\nu} \hat{\sigma}_{2}\right) \Delta_{\boldsymbol{k}}^{*}\left(\hat{\sigma}_{\nu} \hat{\sigma}_{2}\right)^{\dagger} \gamma\left(i \hat{\sigma}_{\nu} \hat{\sigma}_{2}\right)=0,
\end{gathered}
$$

where we have used

$$
\begin{aligned}
\hat{\sim}_{k} & =\left[\hat{\Delta}_{-k}\right]^{*}=\left[\hat{\Delta}_{-k}^{T}\right]^{\dagger}=\left[-\hat{\Delta}_{k}\right]^{\dagger}=\left[-\Delta_{k}\left(\hat{\sigma}_{\nu} \hat{\sigma}_{2}\right)^{\dagger}\right] \\
& =-\Delta_{k}^{*}\left(\hat{\sigma}_{\nu} \hat{\sigma}_{2}\right)^{\dagger}
\end{aligned}
$$

and $\hat{\Delta}_{-\boldsymbol{k}}^{T}=-\hat{\Delta}_{\boldsymbol{k}}$ follows from the Pauli principle. Using $\left(i \hat{\sigma}_{\nu} \hat{\sigma}_{2}\right)\left(i \hat{\sigma}_{\nu} \hat{\sigma}_{2}\right)^{\dagger}=\hat{\sigma}_{0}$ we obtain Eq. (7).

\section{APPENDIX B: BARRIER-POTENTIAL DEPENDENCE OF THE INDUCED MAGNETIZATION}

In this Appendix, we discuss the effects of the barrier potential $z_{0}$ that changes reflection coefficient. The $z_{0}$ dependencies of $\left.M\right|_{x=0^{+}}$for the one-dimensional FM/SC models are shown in Fig. 11. The induced magnetization is not a monotonic function of the barrier parameter $z_{0}$. When $z_{0} \rightarrow \infty$, the reflection coefficient (18) becomes spin-independent (i.e., $\left.r_{\alpha} \rightarrow-1\right)$. Therefore, $M_{x=0^{+}}$vanishes in this limit. When $z_{0}=0$, the reflection coefficients in Eq. (18) are real as long as $k_{\alpha}$ is real (i.e., $\mathrm{F}$ is a ferromagnetic metal), which means that the reflected quasiparticles do not have an additional spin-dependent phase shift. As a result, no magnetization is

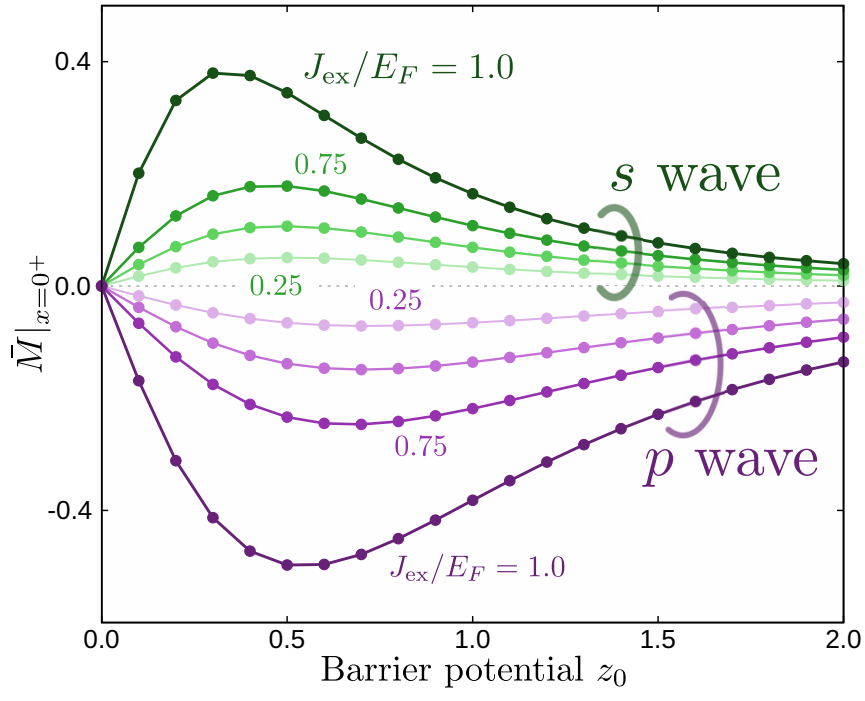

FIG. 11. Induced magnetization at the interface of a $1 D \mathrm{FM} / \mathrm{SC}$ model. The magnetization $\left.M\right|_{x=0^{+}}$for $s$ - and $p$-wave superconductors are respectively positive and negative regardless of the magnitude of $z_{0}$. The temperature is set to $T=0.2 T_{c}$.

induced in the SC. Note that we have confirmed $\theta_{\mathrm{SM}}<0$ for all set of $z_{0}$ and $J_{\text {ex }}$.

\section{APPENDIX C: INDUCED MAGNETIZATION AND ANDREEV BOUND STATES}

We discuss the relation between the direction of the induced magnetization and the Andreev bound states, where we assume magnetic wall (i.e., $\left|r_{\alpha}\right|=1$ ).

The diagonal part of the Green's function is given by Eq. (24). Using the boundary condition (21), we have the diagonal part of the Green's function

$$
\hat{g}_{ \pm k}=\left(1-\gamma_{-k} \underline{\gamma}_{+k} e^{i \hat{\sigma}_{3} \theta_{\mathrm{SM}}}\right)^{-1}\left(1+\gamma_{-k} \underline{\gamma}_{+k} e^{i \hat{\sigma}_{3} \theta_{\mathrm{SM}}}\right),
$$

where we have used $\hat{\Gamma}_{-\hat{\sim}_{+}}=\hat{\gamma}_{-} \hat{\sim}_{+}=\gamma_{-} \underline{\gamma}_{+} e^{i \hat{\sigma}_{3} \theta_{\mathrm{SM}}}$. In Eq. (C1), we have used that the reflection coefficients in the magnetic-wall model can be given by

$$
\hat{r}=\exp \left[i \theta_{\mathrm{SM}} \hat{\sigma}_{3} / 2\right] \text {. }
$$

In what follows, we will make the subscript $\pm k$ implicit because the Green's function at the interface does not depend on the direction of motion. The Green's function can be reduced to

$$
\begin{gathered}
\hat{g}=\frac{1}{\Xi}\left[\left(1-\mathcal{R}^{2}\right) \hat{\sigma}_{0}+2 i \hat{\sigma}_{3} \mathcal{R} \sin \theta_{\mathrm{SM}}\right], \\
\Xi=\left(1-\mathcal{R} \cos \theta_{\mathrm{SM}}\right)^{2}+\mathcal{R}^{2} \sin ^{2} \theta_{\mathrm{SM}},
\end{gathered}
$$

where $\mathcal{R}=\gamma_{-k} \underline{\gamma}_{+k}$. Assuming $\theta_{\mathrm{SM}} \ll 1$, we have

$$
\begin{gathered}
\hat{g} \approx g^{(0)} \hat{\sigma}_{0}+g^{(1)} \hat{\sigma}_{3}, \\
g^{(0)}=\frac{1+\mathcal{R}}{1-\mathcal{R}}, \quad g^{(1)}=\frac{2 i \mathcal{R} \theta_{\mathrm{SM}}}{(1-\mathcal{R})^{2}},
\end{gathered}
$$

where $g^{(0)}$ is the unperturbed Green's function and $g^{(1)}$ is the linear term with respect to $\theta_{\mathrm{SM}}$. In the $1 \mathrm{D}$ limit, the induced 
magnetization at the interface can be calculated from $g^{(1)}$ :

$$
\left.M\right|_{x=0}=4 \pi \mu_{B} N_{0} T \sum_{n=0}^{n_{c}} \operatorname{Im}\left[g^{(1)}\right] .
$$

Substituting $g^{(1)}$, we have

$$
\begin{gathered}
\left.\bar{M}\right|_{x=0}=\frac{T}{T_{c}} \sum_{n=0}^{n_{c}} \frac{4 s_{p} \Delta_{0}^{2} \theta_{\mathrm{SM}}}{\left[\left(1-s_{p}\right) \omega_{n}+\left(1+s_{p}\right) \sqrt{\omega_{n}^{2}+\Delta_{0}^{2}}\right]^{2}}, \\
s_{p}=\Delta_{+k} \Delta_{-k} / \Delta_{0}^{2}=\operatorname{sgn}\left[\Delta_{+k} \Delta_{-k}\right],
\end{gathered}
$$

where $\bar{M}=M / 2 \pi \mu_{B} N_{0} T_{c}$ and we have used

$$
\mathcal{R}=s_{p} \frac{\omega_{n}-\sqrt{\omega_{n}^{2}+\Delta_{0}^{2}}}{\omega_{n}+\sqrt{\omega_{n}^{2}+\Delta_{0}^{2}}},
$$

which is valid under the uniform pair potential. We see from Eq. (C8) that the direction of the induced magnetization $\left.\bar{M}\right|_{x=0}$ is determined by the factor $s_{p}$, which reflects the pairing symmetry of the SC: $s_{p}=+1$ for the $s^{-}, p_{y^{-}}$, and $d_{x^{2}-y^{2}}$-wave pairings and $s_{p}=-1$ for $p_{x}$-and $d_{x y}$-wave SCs. The condition for the ABS in terms of $s_{p}$ is simply given by $s_{p}=-1$. Namely, $\left.\bar{M}\right|_{x=0}$ is determined by whether ABS is present or not (i.e., $s_{p}=-1$ or +1 ). Moreover, the induced magnetization for $s_{p}=-1$ is enhanced at low temperature because its energy dependence is $\left.M\right|_{x=0} \sim 1 / \omega_{n}$.

\section{APPENDIX D: SYMMETRY OF COOPER PAIRS AND INDUCED MAGNETIZATION}

When there is a spin-dependent potential, subdominant pairing component must be induced because of the symmetry breaking. Near the interface of an F/SC junction, the anomalous Green functions are expressed as a superposition of the spin-triplet and singlet pairs:

$$
\begin{gathered}
\hat{g}=\operatorname{diag}\left[g_{\uparrow}, g_{\downarrow}\right], \quad \hat{f}=f_{0} i \hat{\sigma}_{2}+f_{3} \hat{\sigma}_{1}, \\
\underset{\sim}{\hat{f}}=\underline{f}_{0}\left(-i \hat{\sigma}_{2}\right)+\underline{f}_{3} \hat{\sigma}_{1},
\end{gathered}
$$

where we consider a spin-dependent potential parallel to the spin quantization axis. From the normalization condition (i.e., $\hat{g}^{2}-\hat{f} \hat{f}=\hat{\sigma}_{0}$ ), we have the explicit forms for $g_{\uparrow}$ and $g_{\downarrow}: g_{\uparrow(\downarrow)}^{2} \stackrel{\sim}{=}=\left[1+f_{0} \underline{f}_{0}+f_{3} \underline{f}_{3}\right]+(-)\left[f_{0} \underline{f}_{3}+f_{3} \underline{f}_{0}\right]$. When $T \sim T_{c}$, the pair amplitude is sufficiently small. Accordingly, the approximated Green's function and the magnetization are given by the following expressions:

$$
\begin{aligned}
& g_{\uparrow(\downarrow)}=1+\frac{1}{2}\left\{\left[f_{0} \underline{f}_{0}+f_{3} \underline{f}_{3}\right]+(-)\left[f_{0} \underline{f}_{3}+f_{3} \underline{f}_{0}\right]\right\} \\
& M(x) \approx 2 \pi \mu_{B} N_{0} T \sum_{\omega_{n}>0} \int_{-\pi}^{\pi} \operatorname{Im}\left[f_{0} \underline{f}_{3}+f_{3} \underline{f}_{0}\right] \frac{d \varphi}{2 \pi} .
\end{aligned}
$$

In a $2 \mathrm{D}$ system, the anomalous Green's function can be expanded in a Fourier series:

$$
f_{v}=\frac{C_{v, 0}}{\sqrt{2 \pi}}+\frac{1}{\sqrt{\pi}} \sum_{l>0}\left[C_{v, l} \cos (l \varphi)+S_{v, l} \sin (l \varphi)\right],
$$

where $C_{v, n}$ are $S_{v, n}$ are coefficients that represent each pairing amplitude (e.g., $C_{\nu=0, l=0}, C_{3,1}$, and $S_{3,2}$ correspond to the $s$-wave spin-singlet, $p_{x}$-wave spin-triplet, and $d_{x y}$-wave spin-triplet pairs). Note that the spin-singlet odd-parity and spin-triplet even-parity components should be odd functions with respect to the Matsubara frequency. In other words, they represent the odd-frequency pair amplitudes. Using $f\left(x, \varphi, i \omega_{n}\right)=f^{*}\left(x, \varphi+\pi, i \omega_{n}\right)$ and the orthogonality of the trigonometric functions, we can obtain

$$
\begin{aligned}
M(x)= & 4 \pi \mu_{B} N_{0} T \sum_{\omega_{n}>0} \operatorname{Im}\left[C_{0,0} C_{3,0}^{*}\right. \\
& \left.+\sum_{l>0}(-1)^{l}\left(C_{0, l} C_{3, l}^{*}+S_{0, l} S_{3, l}^{*}\right)\right] .
\end{aligned}
$$

In the $1 \mathrm{D}$ limit, in particular, the magnetization is given by

$$
M=4 \pi \mu_{B} N_{0} T \sum_{\omega_{n}>0} \operatorname{Im}\left[f_{0, \mathrm{SW}} f_{3, \mathrm{SW}}^{*}+f_{0, \mathrm{PW}} f_{3, \mathrm{PW}}^{*}\right],
$$

where SW and PW stand for $s$ - and $p$-wave pairings (i.e., even-parity and odd-parity pairing in the 1D limit). The magnetization is generated by the product of the spin-singlet and spin-triplet pairs.

\section{APPENDIX E: SPIN-MIXING ANGLE AND FERMI SURFACES}

The sign of the spin-mixing angle $\theta_{\mathrm{SM}}$ is not simply determined by whether the F is an FM or HM [39]. We show the evolution of the Fermi surfaces in Fig. 12, where the Fermi energies are set to $E_{F}=E_{S}$. The magnetization is set to (a) $J_{\text {ex }}=0.5 E_{F}$, (b) $0.9 E_{F}$, and (c) $1.1 E_{F}$. Increasing $J_{\text {ex }}$, the spin bands split in the F. As a result, there is only one Fermi surface for the channels with $k_{\|}$as shown in the green region in Fig. 12. When $J_{\text {ex }}>E_{F}$, the Fermi surface for the down-spin band vanishes. Comparing Figs. 12 and 10(f), we see that $\operatorname{sgn}\left[\theta_{\mathrm{SM}}\right]$ is not in an obvious way related to the band structure in the F.

In junctions of a ferromagnet and a normal metal, the spin-dependent potentials in the magnet give rise to a phase delay of the wave function for the reflected quasiparticle [39]. The quasiparticle injected from the normal metal penetrates into the ferromagnet even when the process is classically forbidden. The quasiparticle is reflected after experiencing the

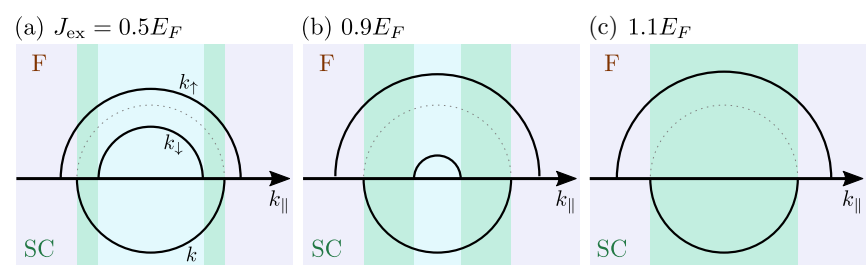

FIG. 12. Evolution of the Fermi surfaces. The Fermi surfaces for the $\mathrm{F}$ and $\mathrm{S}$ are plotted in the upper and lower half plane in each figure. The Fermi wave numbers of up- and down-spin bands are denoted by $k_{\uparrow}$ and $k_{\downarrow}$, whereas that for the SC is denoted by $k$. The exchange energy is set to (a) $J_{\text {ex }}=0.5 E_{F}$, (b) $0.9 E_{F}$, and (c) $1.1 E_{F}$. There are two Fermi surfaces in the $\mathrm{F}$ in the light-blue region, whereas only one Fermi surface exists in the green region. The outer light-purple region is irrelevant to the IPE. 
spin-dependent potential, which results in an additional phase. Therefore, the spin-mixing angle is not only determined by the electronic structure in the ferromagnet but by how the quasiparticle experiences the magnetic potentials at the interface.
[1] J. Bardeen, L. N. Cooper, and J. R. Schrieffer, Theory of superconductivity, Phys. Rev. 108, 1175 (1957).

[2] L. J. Buchholtz and G. Zwicknagl, Identification of p-wave superconductors, Phys. Rev. B 23, 5788 (1981).

[3] J. Hara and K. Nagai, A polar state in a slab as a soluble model of p-wave Fermi superfluid in finite geometry, Prog. Theor. Phys. 76, 1237 (1986).

[4] C.-R. Hu, Midgap Surface States as a Novel Signature For $d_{x a}^{2}$ $x_{b}^{2}$-Wave Superconductivity, Phys. Rev. Lett. 72, 1526 (1994).

[5] Y. Tanaka and S. Kashiwaya, Theory of Tunneling Spectroscopy of $d$-Wave Superconductors, Phys. Rev. Lett. 74, 3451 (1995).

[6] S. Kashiwaya and Y. Tanaka, Tunnelling effects on surface bound states in unconventional superconductors, Rep. Prog. Phys. 63, 1641 (2000).

[7] T. Löfwander, V. S. Shumeiko, and G. Wendin, Andreev bound states in high- $T_{\mathrm{c}}$ superconducting junctions, Supercond. Sci. Technol. 14, R53 (2001).

[8] Y. Tanaka and S. Kashiwaya, Theory of Josephson effects in anisotropic superconductors, Phys. Rev. B 56, 892 (1997).

[9] Y. Asano, Disappearance of ensemble-averaged Josephson current in dirty superconductor-normal-superconductor junctions of $d$-wave superconductors, Phys. Rev. B 64, 014511 (2001).

[10] M. Eschrig, J. Kopu, J. C. Cuevas, and G. Schön, Theory of Half-Metal/Superconductor Heterostructures, Phys. Rev. Lett. 90, 137003 (2003).

[11] Y. Tanaka and S. Kashiwaya, Anomalous charge transport in triplet superconductor junctions, Phys. Rev. B 70, 012507 (2004).

[12] Y. Asano, Y. Tanaka, and S. Kashiwaya, Phenomenological theory of zero-energy Andreev resonant states, Phys. Rev. B 69, 134501 (2004).

[13] H.-J. Kwon, K. Sengupta, and V. M. Yakovenko, Fractional ac Josephson effect in $p$-and $d$-wave superconductors, Eur. Phys. J. B 37, 349 (2004).

[14] M. Krawiec, B. L. Györffy, and J. F. Annett, Current-carrying Andreev bound states in a superconductor-ferromagnet proximity system, Phys. Rev. B 70, 134519 (2004).

[15] J. Kopu, M. Eschrig, J. C. Cuevas, and M. Fogelström, Transfer-matrix description of heterostructures involving superconductors and ferromagnets, Phys. Rev. B 69, 094501 (2004).

[16] M. Eschrig, J. Kopu, A Konstandin, J. C. Cuevas, M. Fogelström, and G. Schön, Singlet-triplet mixing in superconductor-ferromagnet hybrid devices, Adv. Solid State Phys. 44, 533 (2004).

[17] A. I. Buzdin, Proximity effects in superconductor-ferromagnet heterostructures, Rev. Mod. Phys. 77, 935 (2005).

[18] F. S. Bergeret, A. F. Volkov, and K. B. Efetov, Odd triplet superconductivity and related phenomena in superconductorferromagnet structures, Rev. Mod. Phys. 77, 1321 (2005).

[19] Y. Asano, Y. Tanaka, T. Yokoyama, and S. Kashiwaya, Josephson current through superconductor/diffusive-normalmetal/superconductor junctions: Interference effects governed by pairing symmetry, Phys. Rev. B 74, 064507 (2006).
[20] Y. Asano, Y. Tanaka, A. A. Golubov, and S. Kashiwaya, Conductance Spectroscopy of Spin-Triplet Superconductors, Phys. Rev. Lett. 99, 067005 (2007).

[21] R. S. Keizer, S. T. B. Goennenwein, T. M. Klapwijk, G. Miao, G. Xiao, and A. Gupta, A spin triplet supercurrent through the half-metallic ferromagnet $\mathrm{CrO}_{2}$, Nature (London) 439, 825 (2006).

[22] M. Eschrig and T. Löfwander, Triplet supercurrents in clean and disordered half-metallic ferromagnets, Nat. Phys. 4, 138 (2008).

[23] Y. Asano, Y. Tanaka, and A. A. Golubov, Josephson Effect Due to Odd-Frequency Pairs in Diffusive Half Metals, Phys. Rev. Lett. 98, 107002 (2007).

[24] V. Braude and Yu. V. Nazarov, Fully Developed Triplet Proximity Effect, Phys. Rev. Lett. 98, 077003 (2007).

[25] M. Houzet and A. I. Buzdin, Long range triplet Josephson effect through a ferromagnetic trilayer, Phys. Rev. B 76, 060504(R) (2007).

[26] A. V. Galaktionov, M. S. Kalenkov, and A. D. Zaikin, Josephson current and Andreev states in superconductorhalf metal-superconductor heterostructures, Phys. Rev. B 77, 094520 (2008).

[27] E. Zhao and J. A. Sauls, Theory of nonequilibrium spin transport and spin-transfer torque in superconducting-ferromagnetic nanostructures, Phys. Rev. B 78, 174511 (2008).

[28] M. S. Kalenkov and A. D. Zaikin, Nonlocal spin-sensitive electron transport in diffusive proximity heterostructures, Phys. Rev. B 82, 024522 (2010).

[29] T. Löfwander, R. Grein, and M. Eschrig, Is $\mathrm{CrO}_{2}$ Fully Spin Polarized? Analysis of Andreev Spectra and Excess Current, Phys. Rev. Lett. 105, 207001 (2010).

[30] G. Metalidis, M. Eschrig, R. Grein, and G. Schön, Nonlocal conductance via overlapping Andreev bound states in ferromagnet-superconductor heterostructures, Phys. Rev. B 82, 180503(R) (2010).

[31] M. Eschrig, Spin-polarized supercurrents for spintronics, Phys. Today 64, 43 (2011).

[32] Y. Tanaka, M. Sato, and N. Nagaosa, Symmetry and topology in superconductors-Odd-frequency pairing and edge states, J. Phys. Soc. Jpn. 81, 011013 (2012).

[33] P. Machon, M. Eschrig, and W. Belzig, Nonlocal Thermoelectric Effects And Nonlocal Onsager Relations in a ThreeTerminal Proximity-Coupled Superconductor-Ferromagnet Device, Phys. Rev. Lett. 110, 047002 (2013).

[34] P. Machon, M. Eschrig, and W. Belzig, Giant thermoelectric effects in a proximity-coupled superconductor-ferromagnet device, New J. Phys. 16, 073002 (2014).

[35] A. Ozaeta, P. Virtanen, F. S. Bergeret, and T. T. Heikkilä, Predicted Very Large Thermoelectric Effect In FerromagnetSuperconductor Junctions in the Presence of a Spin-Splitting Magnetic Field, Phys. Rev. Lett. 112, 057001 (2014).

[36] A. Di Bernardo, S. Diesch, Y. Gu, J. Linder, G. Divitini, C. Ducati, E. Scheer, M. G. Blamire, and J. W. A. Robinson, Signature of magnetic-dependent gapless odd frequency states at 
superconductor/ferromagnet interfaces, Nat. Commun. 6, 8053 (2015).

[37] J. Linder and J. W. A. Robinson, Superconducting spintronics, Nat. Phys. 11, 307 (2015).

[38] M. Eschrig, Spin-polarized supercurrents for spintronics: A review of current progress, Rep. Prog. Phys. 78, 104501 (2015).

[39] M. Eschrig, Theory of Andreev bound states in S-F-S junctions and S-F proximity devices, Phil. Trans. R. Soc. A 376, 20150149 (2018).

[40] S.-I. Suzuki, M. Sato, and Y. Tanaka, Identifying possible pairing states in $\mathrm{Sr}_{2} \mathrm{RuO}_{4}$ by tunneling spectroscopy, Phys. Rev. B 101, 054505 (2020).

[41] S. Higashitani, Mechanism of paramagnetic Meissner effect in high-temperature superconductors, J. Phys. Soc. Jpn. 66, 2556 (1997).

[42] Y. Tanaka, Y. Asano, A. A. Golubov, and S. Kashiwaya, Anomalous features of the proximity effect in triplet superconductors, Phys. Rev. B 72, 140503(R) (2005).

[43] Y. Asano, A. A. Golubov, Y. V. Fominov, and Y. Tanaka, Unconventional Surface Impedance of a Normal-Metal Film Covering a Spin-Triplet Superconductor Due to Odd-Frequency Cooper Pairs, Phys. Rev. Lett. 107, 087001 (2011).

[44] T. Yokoyama, Y. Tanaka, and N. Nagaosa, Anomalous Meissner Effect in a Normal-Metal-Superconductor Junction With a Spin-Active Interface, Phys. Rev. Lett. 106, 246601 (2011).

[45] S.-I. Suzuki and Y. Asano, Paramagnetic instability of small topological superconductors, Phys. Rev. B 89, 184508 (2014).

[46] A. Di Bernardo, Z. Salman, X. L. Wang, M. Amado, M. Egilmez, M. G. Flokstra, A. Suter, S. L. Lee, J. H. Zhao, T. Prokscha, E. Morenzoni, M. G. Blamire, J. Linder, and J. W. A. Robinson, Intrinsic Paramagnetic Meissner Effect Due to $s$-Wave Odd-Frequency Superconductivity, Phys. Rev. X 5, 041021 (2015).

[47] S.-I. Suzuki and Y. Asano, Effects of surface roughness on the paramagnetic response of small unconventional superconductors, Phys. Rev. B 91, 214510 (2015).

[48] Y. Asano and A. Sasaki, Odd-frequency Cooper pairs in twoband superconductors and their magnetic response, Phys. Rev. B 92, 224508 (2015).

[49] S.-I. Suzuki and Y. Asano, Spontaneous edge current in a small chiral superconductor with a rough surface, Phys. Rev. B 94, 155302 (2016).

[50] S.-P. Lee, R. M. Lutchyn, and J. Maciejko, Odd-frequency superconductivity in a nanowire coupled to Majorana zero modes, Phys. Rev. B 95, 184506 (2017).

[51] J. A. Krieger, A. Pertsova, S. R. Giblin, M. Döbeli, T. Prokscha, C. W. Schneider, A. Suter, T. Hesjedal, A. V. Balatsky, and Z. Salman, Proximity-Induced Odd-Frequency Superconductivity in a Topological Insulator, Phys. Rev. Lett. 125, 026802 (2020).

[52] S. Higashitani, H. Takeuchi, S. Matsuo, Y. Nagato, and K. Nagai, Magnetic Response of Odd-Frequency $s$-Wave Cooper Pairs in a Superfluid Proximity System, Phys. Rev. Lett. 110, 175301 (2013).

[53] S. Higashitani, Odd-frequency pairing effect on the superfluid density and the Pauli spin susceptibility in spatially nonuniform spin-singlet superconductors, Phys. Rev. B 89, 184505 (2014).

[54] G. Deutscher and P. G. De Gennes, Superconductivity (Marcel Dekker, New York, 1969), pp. 1005-34.

[55] A. F. Volkov, A. V. Zaitsev, and T. M. Klapwijk, Proximity effect under nonequilibrium conditions in double-barrier su- perconducting junctions, Physica C: Superconductivity 210, 21 (1993).

[56] A. A. Golubov and M. Yu. Kupriyanov, Theoretical investigation of Josephson tunnel junctions with spatially inhomogeneous superconducting electrodes, J. Low Temp. Phys. 70, 83 (1988).

[57] A. A. Golubov and M. Yu. Kupriyanov, Josephson effect in SNINS and SNIN tunnel structures with finite transparency of the sn boundaries, Zh. Èksp. Teor. Fiz. 96, 1420 (1989) [Sov. Phys. JETP 69, 805 (1989)]).

[58] A. A. Golubov, F. K. Wilhelm, and A. D. Zaikin, Coherent charge transport in metallic proximity structures, Phys. Rev. B 55, 1123 (1997).

[59] S.-I. Suzuki, A. A. Golubov, Y. Asano, and Y. Tanaka, Effects of phase coherence on local density of states in superconducting proximity structures, Phys. Rev. B 100, 024511 (2019).

[60] Y. Tanaka, Y. Tanuma, and A. A. Golubov, Odd-frequency pairing in normal-metal/superconductor junctions, Phys. Rev. B 76, 054522 (2007).

[61] V. L. Berezinskii, New model of the anisotropic phase of superfluid ${ }^{3} \mathrm{He}$, Zh. Eksp. Teor. Fiz. 20, 628 (1974) [JETP Lett. 20, 287 (1974)].

[62] A. Balatsky and E. Abrahams, New class of singlet superconductors which break the time reversal and parity, Phys. Rev. B 45, 13125 (1992).

[63] Ya. V. Fominov, Y. Tanaka, Y. Asano, and M. Eschrig, Odd-frequency superconducting states with different types of Meissner response: Problem of coexistence, Phys. Rev. B 91, 144514 (2015).

[64] T. Tokuyasu, J. A. Sauls, and D. Rainer, Proximity effect of a ferromagnetic insulator in contact with a superconductor, Phys. Rev. B 38, 8823 (1988).

[65] F. S. Bergeret, A. F. Volkov, and K. B. Efetov, Induced ferromagnetism due to superconductivity in superconductorferromagnet structures, Phys. Rev. B 69, 174504 (2004).

[66] S. Tollis, M. Daumens, and A. Buzdin, Inversion of the proximity effect in atomic-scale ferromagnet/superconductor/ ferromagnet trilayers, Phys. Rev. B 71, 024510 (2005).

[67] F. S. Bergeret, A. Levy Yeyati, and A. Martín-Rodero, Inverse proximity effect in superconductor-ferromagnet structures: From the ballistic to the diffusive limit, Phys. Rev. B 72, 064524 (2005).

[68] R. I. Salikhov, I. A. Garifullin, N. N. Garif'yanov, L. R Tagirov, K. Theis-Bröhl, K. Westerholt, and H. Zabel, Experimental Observation of the Spin Screening Effect in Superconductor/Ferromagnet Thin Film Heterostructures, Phys. Rev. Lett. 102, 087003 (2009).

[69] J. Xia, V. Shelukhin, M. Karpovski, A. Kapitulnik, and A. Palevski, Inverse Proximity Effect in SuperconductorFerromagnet Bilayer Structures, Phys. Rev. Lett. 102, 087004 (2009).

[70] R. Grein, T. Löfwander, and M. Eschrig, Inverse proximity effect and influence of disorder on triplet supercurrents in strongly spin-polarized ferromagnets, Phys. Rev. B 88, 054502 (2013).

[71] V. Yagovtsev, N. Pugach, and M. Eschrig, The inverse proximity effect in strong ferromagnet-superconductor structures, Supercond. Sci. Technol. 34, 025003 (2021).

[72] Th. Mühge, Yu. V. Goryunov, K. Theis-Bröhl, K. Westerholt, H. Zabel, and I. A. Garifullin, Proximity effect in superconductor/ferromagnet layered system: Influence of superconductivity 
on magnetic properties of nb/fe epitaxial bilayers, Appl. Magn. Reson. 14, 567 (1998).

[73] I. A. Garifullin, D. A. Tikhonov, N. N. Garifyanov, M. Z. Fattakhov, K. Theis-Bröhl, K. Westerholt, and H. Zabel, Possible reconstruction of the ferromagnetic state under the influence of superconductivity in epitaxial $\mathrm{V} / \mathrm{Pd}_{1-\mathrm{x}} \mathrm{Fe}_{\mathrm{x}}$ bilayers, Appl. Magn. Reson. 22, 439 (2002).

[74] N. Schopohl and K. Maki, Quasiparticle spectrum around a vortex line in a $d$-wave superconductor, Phys. Rev. B 52, 490 (1995).

[75] M. Eschrig, J. A. Sauls, and D. Rainer, Electromagnetic response of a vortex in layered superconductors, Phys. Rev. B 60, 10447 (1999).

[76] M. Eschrig, Distribution functions in nonequilibrium theory of superconductivity and Andreev spectroscopy in unconventional superconductors, Phys. Rev. B 61, 9061 (2000).

[77] M. Eschrig, Scattering problem in nonequilibrium quasiclassical theory of metals and superconductors: General boundary conditions and applications, Phys. Rev. B 80, 134511 (2009).

[78] M. Eschrig, A. Cottet, W. Belzig, and J. Linder, General boundary conditions for quasiclassical theory of superconductivity in the diffusive limit: application to strongly spin-polarized systems, New J. Phys. 17, 083037 (2015).

[79] The anomalous Green's functions $\hat{f}$ describes the existence of the Cooper pairs, whereas the Green's function $\hat{g}$ describes the quasiparticle. We can describe both of them by the coherence function $\hat{\gamma}$. The coherence function $\hat{\gamma}$ has more similarities to $\hat{f}$ than to $\hat{g}$ [e.g., $\hat{\gamma}=0$ and $\hat{f}=0$ when $\hat{\Delta} \rightarrow 0$ ] since it is proportional to $\hat{f}$.

[80] In the present case (i.e., magnetic potentials are parallel to the spin quantization axis), the reflection coefficients are diagonal in spin space (i.e., $\hat{r}=\operatorname{diag}\left[r_{\uparrow}, r_{\downarrow}\right]$ ).
[81] M. Ashida, S. Aoyama, J. Hara, and K. Nagai, Green ‘s function in proximity-contact superconducting-normal double layers, Phys. Rev. B 40, 8673 (1989).

[82] Y. Nagato, K. Nagai, and J. Hara, Theory of the Andreev reflection and the density of states in proximity contact normalsuperconducting infinite double-layer, J. Low Temp. Phys. 93, 33 (1993).

[83] Y. Tanuma, Y. Tanaka, and S. Kashiwaya, Tunneling conductance of normal metal $/ d_{x^{2}-y^{2}}$-wave superconductor junctions in the presence of broken time-reversal symmetry states near interfaces, Phys. Rev. B 64, 214519 (2001).

[84] T. Hirai, Y. Tanaka, N. Yoshida, Y. Asano, J. Inoue, and S. Kashiwaya, Temperature dependence of spin-polarized transport in ferromagnet/unconventional superconductor junctions, Phys. Rev. B 67, 174501 (2003).

[85] The ferromagnet $(F)$ is classified into the three classes depending on the number of the Fermi surfaces: the ferromagnetic metal, half metal, and ferromagnetic insulator have two, one, and zero Fermi surface(s), respectively. These materials can be modeled by tuning the Fermi energy in the $\mathrm{F} E_{F}$ and the exchange energy $J_{\text {ex }}$. To sweep this parameter space of $E_{F}$ and $J_{\text {ex }}$, we fix $E_{F}$ and change $J_{\text {ex }}$.

[86] The spin-mixing angle in Ref. [64] is defined with an extra minus sign compared with ours.

[87] S. Higashitani, Spin current as a manifestation of surface oddfrequency pairing in superfluid ${ }^{3} \mathrm{He}$, J. Phys. Soc. Jpn. 83, 075002 (2014).

[88] M. Eschrig, T. Löfwander, T. Champel, J. C. Cuevas, J. Kopu, and G. Schön, Symmetries of pairing correlations in superconductor-ferromagnet nanostructures, J. Low Temp. Phys. 147, 457 (2007). 